\title{
Refinement multidimensional dynamic inequalities with general kernels and measures
}

\author{
Samir H. Saker ${ }^{1}$, Haytham M. Rezk², Islam Abohela ${ }^{3}$ and Dumitru Baleanu 4, $^{*}$ (])
}

\section{"Correspondence:}

dumitru@cankaya.edu.tr

${ }^{4}$ Department of Mathematics,

Cankaya University, Ankara, Turkey

${ }^{5}$ Institute of Space Sciences,

Magurele-Bucharest, Romania

Full list of author information is

available at the end of the article

\begin{abstract}
Using the properties of superquadratic and subquadratic functions, we establish some new refinement multidimensional dynamic inequalities of Hardy's type on time scales. Our results contain some of the recent results related to classical multidimensional Hardy's and Pólya-Knopp's inequalities on time scales. To show motivation of the paper, we apply our results to obtain some particular multidimensional cases and provide refinements of some Hardy-type inequalities known in the literature.
\end{abstract}

Keywords: Hardy-type inequality; Pólya-Knopp-type inequalities; Superquadratic (subquadratic) functions; Hardy's integral operator with kernel; Time scales

\section{Introduction}

Hardy [13] proved the integral inequality

$$
\int_{0}^{\infty}\left(\frac{1}{t} \int_{0}^{t} g(s) d s\right)^{p} d t \leq\left(\frac{p}{p-1}\right)^{p} \int_{0}^{\infty} g^{p}(t) d t
$$

for a nonnegative integrable function $g$ in the space $L^{p}\left(R_{+}\right), 1<p<\infty$, where the constant $(p /(p-1))^{p}$ is the best possible. Rewriting (1) with the function $g^{1 / p}$ instead of $g$ and taking the limit as $p \rightarrow \infty$, we get the limiting case of the Hardy inequality, known as the PólyaKnopp inequality (see [15]),

$$
\int_{0}^{\infty} \exp \left(\frac{1}{t} \int_{0}^{t} \ln g(s) d s\right) d t \leq e \int_{0}^{\infty} g(t) d t
$$

for positive functions $g \in L^{1}\left(R_{+}\right)$, where the constant $e$ is the best possible. Recently, Kaijser et al. [14] have pointed out that both (1) and (2) are just particular cases of the much more general Hardy-Knopp's inequality

$$
\int_{0}^{\infty} \Psi\left(\frac{1}{t} \int_{0}^{t} g(s) d s\right) \frac{d t}{t} \leq \int_{0}^{\infty} \Psi(g(t)) \frac{d t}{t}
$$

for convex functions $\Psi$ on $R_{+}$and locally integrable positive functions $g: R_{+} \rightarrow R_{+}$. The Hardy-type inequalities and their general forms have been studied in the literature in vari-

(c) The Author(s) 2019. This article is distributed under the terms of the Creative Commons Attribution 4.0 International License (http://creativecommons.org/licenses/by/4.0/), which permits unrestricted use, distribution, and reproduction in any medium, provided you give appropriate credit to the original author(s) and the source, provide a link to the Creative Commons license, and indicate if changes were made. 
ous modifications both in the continuous and discrete settings. They have extensive applications to partial differential and difference equations, harmonic analysis, approximations, number theory, optimization, convex geometry, spectral theory of differential and difference operators, and others (see [24-28, 30]). One such approach involves superquadratic functions, which is a class of functions closely connected to convex functions, and various inequalities with different kernels and extensions to multidimensional cases have been obtained. For example, in 1967, Godunova [10] proved the inequality

$$
\int_{\mathbf{R}_{+}^{\mathbf{n}}} \Psi\left(\frac{1}{t_{1} \ldots t_{n}} \int_{\mathbf{R}_{+}^{\mathbf{n}}} l\left(\frac{s_{1}}{t_{1}}, \frac{s_{2}}{t_{2}}, \ldots, \frac{s_{n}}{t_{n}}\right) g(\mathbf{s}) \mathbf{d s}\right) \frac{\mathbf{d t}}{t_{1} \ldots t_{n}} \leq \int_{\mathbf{R}_{+}^{\mathbf{n}}} \frac{\Psi(g(\mathbf{t})) \mathbf{d} \mathbf{t}}{t_{1} \ldots t_{n}}
$$

for nonnegative functions $l: R_{+}^{n} \rightarrow R_{+}$such that $\int_{R_{+}^{n}} l(\mathbf{t}) \mathbf{d t}=1$, convex functions $\Psi$ : $[0, \infty) \rightarrow[0, \infty)$, and nonnegative functions $g$ on $R_{+}^{n}$ such that the function $\Psi(g(\mathbf{t})) /$ $\left(t_{1} \ldots t_{n}\right)$ is integrable on $R_{+}^{n}$.

In 2003, Čižmešija et al. [8] proved a generalization of the Hardy-Knopp inequality (3) with two weighted functions. In particular, they proved that if $0<b \leq \infty, \lambda:(0, b) \rightarrow R$ is a nonnegative function such that the function $t \rightarrow \lambda(t) / t^{2}$ is locally integrable in $(0, b)$, and $\Psi$ is a convex function on $(a, c)$, where $-\infty \leq a \leq c \leq \infty$, then

$$
\int_{0}^{b} \lambda(t) \Psi\left(\frac{1}{t} \int_{0}^{t} g(s) d s\right) \frac{d t}{t} \leq \int_{0}^{b} \eta(t) \Psi(g(t)) \frac{d t}{t}
$$

for all integrable functions $g:(0, b) \rightarrow R$ such that $g(t) \in(a, c)$ for all $t \in(0, b)$, where $\eta(s):=s \int_{s}^{b} \frac{\lambda(t)}{t^{2}} d t$ for $s \in(0, b)$. In 2008, Oguntuase and Persson [17] used the notion of superquadratic and subquadratic functions to obtain a new Hardy-type inequality for $p \geq 2$, which holds in the reverse order for $1<p<2$.

In [18] the authors obtained some new Hardy-type inequalities on time scales by using the notion of superquadratic functions. In particular, they proved that if $\left(\Omega_{1}, \Sigma_{1}, \mu_{1}\right)$ and $\left(\Omega_{2}, \Sigma_{2}, \mu_{2}\right)$ are two time scale measure spaces with positive $\sigma$-finite measures, $\lambda: \Omega_{1} \rightarrow$ $R$ and $k: \Omega_{1} \times \Omega_{2} \rightarrow R$ are nonnegative functions such that $k(t, \cdot)$ is a $\Delta \mu_{2}$-integrable function for $t \in \Omega_{2}, K: \Omega_{1} \rightarrow R$ is defined by $K(t):=\int_{\Omega_{2}} k(t, s) \Delta \mu_{2}(s)<\infty, t \in \Omega_{1}$, and $\eta(s):=\int_{\Omega_{1}} \lambda(t) \frac{k(t, s)}{K(t)} \Delta \mu_{1}(t)<\infty, s \in \Omega_{2}$, then

$$
\begin{aligned}
\int_{\Omega_{1}} \lambda(t) \Psi\left(A_{k} g(t)\right) \Delta \mu_{1}(t) \\
\quad+\int_{\Omega_{1}} \int_{\Omega_{2}} \lambda(t) \frac{k(t, s)}{K(t)} \Psi\left(\left|g(s)-A_{k} g(t)\right|\right) \Delta \mu_{1}(t) \Delta \mu_{2}(s) \\
\leq \int_{\Omega_{2}} \eta(t) \Psi(g(t)) \Delta \mu_{2}(t),
\end{aligned}
$$

for nonnegative $\Delta \mu_{2}$-integrable functions $g: \Omega_{2} \rightarrow R$ and nonnegative superquadratic functions $\Psi:[a, \infty) \rightarrow R(a \geq 0)$, where $A_{k} g: \Omega_{1} \rightarrow R$ is defined by $A_{k} g(t):=\frac{1}{K(t)} \int_{\Omega_{2}} k(t$, $s) g(s) \Delta \mu_{2}(s), t \in \Omega_{1}$. The general idea in this direction is proving a result for a dynamic inequality, where the domain of the unknown function is a so-called time scale $T$, which may be an arbitrary nonempty closed subset of the real numbers $R$. Fore more details of calculus on time scales, we refer to the books $[3,6,7]$. Three most popular examples of calculus on time scales are differential calculus, difference calculus, and quantum calculus, that is, 
when $T=R, T=N$, and $T=q^{N_{0}}=\left\{q^{t}: t \in N_{0}\right\}$ with $q>1$, respectively. A time scale $T$ is an arbitrary nonempty closed subset of the real numbers $R$. We define the time scale interval $[a, b]_{T}$ by $[a, b]_{T}:=[a, b] \cap T$. A function $g: T \rightarrow R$ is said to be right-dense continuous (rd-continuous) if it is continuous at all right-dense points in $T$ and its left-sided finite limits exist at all left-dense points in $T$. The set of all such rd-continuous functions is denoted by $C_{r d}(T):=C_{r d}(T, R)$. The graininess $\mu$ on time scale $T$ is defined by $\mu(t):=\sigma(t)-t$, and for a function $g: T \rightarrow R, g^{\sigma}(t)$ denotes $g(\sigma(t))$, where $\sigma: T \rightarrow T$ is the forward jump operator given by $\sigma(t):=\inf \{s \in T: s>t\}$. The basic formulas involving delta derivatives are the following: $g^{\sigma}:=g+\mu g^{\Delta},(g g)^{\Delta}:=f^{\Delta} g+f^{\sigma} g^{\Delta}:=f g^{\Delta}+f^{\Delta} g^{\sigma}$, and $\left(\frac{f}{g}\right)^{\Delta}:=\frac{f^{\Delta} g-f g^{\Delta}}{g g^{\sigma}}$, where $g, g$ are delta differentiable, and $g g^{\sigma} \neq 0$ in the last formula. Let $T$ be a time scale, and let $[a, b) \subset T$. The Lebesgue integral associated with the measure $\mu$ on $[a, b)$ is called the Lebesgue $\Delta$-integral. If $g:[a, b) \rightarrow R$, then we denote the corresponding $\Delta$-integral of $g$ over $[a, b)$ by $\int_{a}^{b} g(t) \Delta t$. In particular, if $T$ is an arbitrary time scale and the interval $[a, b) \subset T$ contains only isolated points, then $\int_{a}^{b} g(t) \Delta t:=\sum_{t \in[a, b)}(\sigma(t)-t) g(t)$.

In [21] the authors proved the time scale version of (5) given by

$$
\int_{a}^{b} \lambda(t) \Psi\left(\frac{1}{\sigma(t)-a} \int_{a}^{\sigma(t)} g(t) \Delta t\right) \frac{\Delta t}{t-a} \leq \int_{a}^{b} \eta(t) \Psi(g(t)) \frac{\Delta t}{t-a}
$$

where $\lambda \in \mathrm{C}_{r d}([a, b], R)$ is a nonnegative function, and the weight function $\eta$ is defined by $\eta(t):=(t-a) \int_{t}^{b} \frac{\lambda(t)}{(t-a)(\sigma(t)-a)} \Delta t$ for $t \in(a, b), \Psi:(c, d) \rightarrow R$ is a continuous convex function, where $c, d \in R$, and $g \in \mathrm{C}_{r d}([a, b], R)$ is a delta-integrable function such that $g(t) \in(c, d)$ for all $t \in[a, b]$. In [5] (see also [22]) the authors proved that if $\left(\Omega, \mathcal{M}, \mu_{\Delta}\right)$ and $\left(\Lambda, \mathcal{L}, \lambda_{\Delta}\right)$ are two time scale measure spaces, $k: \Omega \times \Lambda \rightarrow R_{+}$is such that $K(t):=\int_{\Lambda} k(t, s) \Delta s<\infty$, $t \in \Omega$, and $\zeta: \Omega \rightarrow R_{+}$is such that $\eta(s):=\int_{\Omega} \frac{k(t, s) \zeta(t)}{K(t)} \Delta t<\infty, s \in \Lambda$, then

$$
\int_{\Omega} \zeta(t) \Psi\left(\frac{1}{K(t)} \int_{\Lambda} k(t, s) g(s) \Delta s\right) \Delta t \leq \int_{\Lambda} \eta(s) \Psi(g(s)) \Delta s
$$

for $\lambda_{\Delta}$-integrable functions $g: \Lambda \rightarrow R$ such that $g(\Lambda) \subset I$ and convex functions $\Psi \in$ $\mathrm{C}(I, R)$, where $I \subset R$.

In recent years, some authors studied the fractional inequalities using the fractional $\mathrm{Ca}$ puto and Riemann-Liouville derivatives. Note that our results can be extended to different types of fractional operators based on fractional calculus [31]. For the advanced development of the fractional calculus without singular kernel of exponential function, we refer the reader to [28]. For the general fractional derivative, we refer the reader to [9, 32]. We also refer the reader to [11,12,23], which give a unification of calculus of the functions on totally disconnected and continuous real line.

In this paper, in the next section, we prove some new refined multidimensional dynamic inequalities of Hardy type with weighted functions and nonnegative kernel using the properties of superquadratic (or subquadratic) functions. Our results contain some recent results related to classical multidimensional Hardy's and Pólya-Knopp's inequalities on time scales. To show motivation of the paper, we will apply our results to obtain some particular multidimensional cases and provide refinements of some Hardy-type inequalities known in the literature. We also discuss some particular cases of the obtained inequalities, related to power and exponential functions, and to the most simplest forms of kernels for illustration. 


\section{Main results}

In this section, we obtain the multidimensional time scale for Hardy-type inequality with general kernel and also with several functions. First, we recall the definition and some basic properties of superquadratic functions, introduced by Abramovich et al. [1] (for more information, see also [2]).

Definition 2.1 A function $\Psi:[0, \infty) \rightarrow R$ is called superquadratic if for all $t \geq 0$, there exists a constant $C_{t} \in R$ such that

$$
\Psi(s)-\Psi(t)-\Psi(|s-t|) \geq C_{t}(s-t) \quad \text { for all } s \geq 0 .
$$

Superquadratic functions are closely related to convex functions. In particular, at the first sight, condition (9) appears to be stronger than convexity. We say that $\Psi$ is subquadratic if $-\Psi$ is superquadratic and the reverse inequality of (9) holds.

Definition 2.2 A function $g:[0, \infty) \rightarrow R$ is superadditive if $g(t+s) \geq g(t)+g(s)$ for all $t$, $s \geq 0$. If the reverse inequality holds, then $g$ is said to be subadditive.

Lemma 2.1 Suppose $\Psi:[0, \infty) \rightarrow R$ is continuously differentiable and such that $\Psi(0) \leq 0$. If $\Psi^{\prime}$ is superadditive, that is, $\Psi^{\prime}(t+s) \geq \Psi^{\prime}(t)+\Psi^{\prime}(s)$ for all $t, s \geq 0$, or the function $t \mapsto \frac{\Psi^{\prime}(t)}{t}$ is nondecreasing on $R_{+}$, then $\Psi$ is superquadratic.

As a consequence, the power function $\Psi:[0, \infty) \rightarrow R, \Psi(t)=t^{p}$, is superquadratic for all $p \in R_{+}, p \geq 2$, and subquadratic for $1<p \leq 2$. Next, we recall a refined Jensen's inequality for superquadratic functions and Minkowski's inequality on time scales, which are used in the proof of the main results.

Lemma 2.2 Let $\left(\Omega, \mathcal{M}, \mu_{\Delta}\right)$ and $\left(\Lambda, \mathcal{L}, \lambda_{\Delta}\right)$ be two finite-dimensional time scale measures spaces. and let $\lambda, \eta$, and $g$ be nonnegative functions on $\Omega, \Lambda$, and $\Omega \times \Lambda$, respectively. If $p \geq 1$, then

$$
\begin{aligned}
& {\left[\int_{\Omega}\left(\int_{\Lambda} g(t, s) \eta(s) d \eta_{\Delta}(s)\right)^{p} \lambda(t) d \mu_{\Delta}(t)\right]^{\frac{1}{p}}} \\
& \quad \leq \int_{\Lambda}\left(\int_{\Omega} g^{p}(t, s) \lambda(t) d \mu_{\Delta}(t)\right) \eta(s) d \eta_{\Delta}(s),
\end{aligned}
$$

provided that all integrals in (10) exist. If $0<p<1$ and

$$
\int_{\Omega}\left(\int_{\Lambda} g \eta d \eta_{\Delta}\right)^{p} \lambda d \mu_{\Delta}>0 \text { and } \int_{\Lambda} g \eta d \eta_{\Delta}>0
$$

then (10) is reversed. For $p<0$, if in addition to (11), $\int_{\Omega} g^{p} \lambda d \mu_{\Delta}>0$, then (10) is also reversed.

In addition, another important characterization of superquadratic functions is the following version of the refined Jensen inequality which is adapted from [4, Theorem 2.5]. 
Lemma 2.3 Let $a, b \in T$ and suppose that $g:[a, b]_{T^{k}} \rightarrow[0, \infty)$ is $r$-continuous and $\Psi$ : $[0, \infty) \rightarrow R$ is continuous and superquadratic. Then

$$
\Psi\left(\frac{1}{b-a} \int_{a}^{b} g(t) \Delta t\right) \leq \frac{1}{b-a} \int_{a}^{b}\left[\Psi(g(s))-\Psi\left(\left|g(s)-\frac{1}{b-a} \int_{a}^{b} g(t) \Delta t\right|\right)\right] \Delta s
$$

For the case $\Psi(t)=t^{p},(12)$ gives the inequality

$$
\left(\frac{1}{b-a} \int_{a}^{b} g(t) \Delta t\right)^{p} \leq \frac{1}{b-a} \int_{a}^{b}\left[g^{p}(s)-\left(\left|g(s)-\frac{1}{b-a} \int_{a}^{b} g(t) \Delta t\right|\right)^{p}\right] \Delta s .
$$

We will further assume (usually without mentioning) that all measures are positive, all functions in the statements of the theorems are nonnegative and measurable, and the integrals considered exist. Before presenting the main results for multidimensional inequalities, it is necessary to introduce some further notations. First, let $n \in Z_{+}$and set $0=(0,0, \ldots, 0), 1=(1,1, \ldots, 1) \in R^{n}$, and for $\lambda=\left(\lambda_{1}, \ldots, \lambda_{n}\right), \eta=\left(\eta_{1}, \ldots, \eta_{n}\right) \in R^{n}$, denote $\frac{\lambda}{\eta}=\left(\frac{\lambda_{1}}{\eta_{1}}, \frac{\lambda_{2}}{\eta_{2}}, \ldots, \frac{\lambda_{n}}{\eta_{n}}\right)$ and $\lambda^{\eta}=\lambda_{1}^{\eta_{1}} \lambda_{2}^{\eta_{2}} \ldots \lambda_{n}^{\eta_{n}}$, and let $\Omega_{1}, \Omega_{2} \subset R^{n}$ be time scale measure spaces. In particular,

$$
\lambda^{\mathbf{1}}=\prod_{i=1}^{n} \lambda_{i}, \quad \lambda^{2}=\left(\prod_{i=1}^{n} \lambda_{i}\right)^{2} \quad \text { and } \quad \lambda^{-\mathbf{1}}=\left(\prod_{i=1}^{n} \lambda_{i}\right)^{-1}
$$

where $\mathbf{n}=\left(n_{1}, \ldots, n_{n}\right)$. Correspondingly, $[\mathbf{a}, \mathbf{b})$ means the set $\left[a_{1}, b_{1}\right) \times\left[a_{2}, b_{2}\right) \times \cdots \times$ $\left[a_{n}, b_{n}\right), \Delta \lambda=\left(\Delta \lambda_{1} \ldots \Delta \lambda_{n}\right)$, and $\lambda^{p}=\left(\lambda_{1} \ldots \lambda_{n}\right)^{p}$. Furthermore, for $\lambda, \eta \in R^{n}$, we write $\lambda<\eta$ if componentwise $\lambda_{i}<\eta_{i}, i=1,2, \ldots, n$, and the relations $\leq, \geq$, and $<$ are defined analogously. Also, for $\mathbf{a}, \mathbf{b} \in R^{n}, \mathbf{a}<\mathbf{b}$, we define $(\mathbf{a}, \mathbf{b})=\left\{\mathbf{t} \in R^{n}: \mathbf{a}<\mathbf{t}<\mathbf{b}\right\}$. Moreover, $(\mathbf{a}, \infty)=\left\{\mathbf{t} \in R^{n}: \mathbf{a}<\mathbf{t}<\infty\right\}$, and the $n$-cells $[\mathbf{a}, \mathbf{b}),(\mathbf{a}, \mathbf{b}]$, and $[\mathbf{a}, \mathbf{b}]$ are defined similarly. Finally, the integral $\int_{[\mathbf{a}, \mathbf{b})} g(\mathbf{t}) \Delta \mathbf{t}$ is interpreted as

$$
\int_{[\mathbf{a}, \mathbf{b})} g(\mathbf{t}) \Delta \mathbf{t}:=\int_{a_{1}}^{b_{1}} \int_{a_{2}}^{b_{2}} \ldots \int_{a_{n}}^{b_{n}} g\left(t_{1}, t_{2}, \ldots, t_{n}\right) \Delta t_{1} \ldots \Delta t_{n} .
$$

In this setting, we can easily prove the following multidimensional time scale Hardy-type inequality with general kernel. We further assume that $\left(\Omega_{1}, \Sigma_{1}, \mu_{1}\right)$ and $\left(\Omega_{2}, \Sigma_{2}, \mu_{2}\right)$ are two time scale measure spaces with positive $\sigma$-finite measures.

Theorem 2.1 Let $\lambda: \Omega_{1} \rightarrow R$ and $k: \Omega_{1} \times \Omega_{2} \rightarrow R$ be nonnegative functions such that $k(t, \cdot)$ is a $\Delta \mu_{2}$-integrable function for $t \in \Omega_{2}$. Suppose that $\mathcal{K}: \Omega_{1} \rightarrow R$ is defined by

$$
\mathbf{0}<K(\mathbf{t}):=\int_{\Omega_{2}} k(\mathbf{t}, \mathbf{s}) \Delta \mu_{2}\left(s_{1}\right) \ldots \Delta \mu_{2}\left(s_{n}\right)<\infty, \quad \mathbf{t} \in \Omega_{1},
$$

and the function $\eta$ be defined by

$$
\eta(\mathbf{s}):=\left(\int_{\Omega_{1}} \lambda(\mathbf{t})\left(\frac{k(\mathbf{t}, \mathbf{s})}{K(\mathbf{t})}\right)^{r} \Delta \mu_{1}\left(t_{1}\right) \ldots \Delta \mu_{1}\left(t_{n}\right)\right)^{\frac{1}{r}}<\infty, \quad \mathbf{s} \in \Omega_{2}
$$


where $r \geq 1$. Furthermore, assume that $g: \Omega_{2} \rightarrow R$ is a nonnegative $\Delta \mu_{2}$-integrable function and define $A_{k} g: \Omega_{1} \rightarrow R$ by

$$
A_{k} g(\mathbf{t}):=\frac{1}{K(\mathbf{t})} \int_{\Omega_{2}} k(\mathbf{t}, \mathbf{s}) g(\mathbf{s}) \Delta \mu_{2}\left(s_{1}\right) \ldots \Delta \mu_{2}\left(s_{n}\right), \quad \mathbf{t} \in \Omega_{1} .
$$

If $\Psi:[\mathbf{a}, \infty)_{T} \rightarrow R\left(a_{i} \geq 0, i=1,2, \ldots, n\right)$ is a nonnegative superquadratic function, then

$$
\begin{aligned}
\int_{\Omega_{1}} & \lambda(\mathbf{t}) \Psi^{r}\left(A_{k} g(\mathbf{t})\right) \Delta \mu_{1}\left(t_{1}\right) \ldots \Delta \mu_{1}\left(t_{n}\right) \\
& +r \int_{\Omega_{1}} \int_{\Omega_{2}} \lambda(\mathbf{t}) \frac{k(\mathbf{t}, \mathbf{s})}{K(\mathbf{t})} \Psi^{r-1}\left(A_{k} g(\mathbf{t})\right) \Psi\left(\left|g(\mathbf{s})-A_{k} g(\mathbf{t})\right|\right) \\
& \times \Delta \mu_{1}\left(t_{1}\right) \ldots \Delta \mu_{1}\left(t_{n}\right) \Delta \mu_{2}\left(s_{1}\right) \ldots \Delta \mu_{2}\left(s_{n}\right) \\
\leq & \left(\int_{\Omega_{2}} \eta(\mathbf{s}) \Psi(g(\mathbf{s})) \Delta \mu_{2}\left(s_{1}\right) \ldots \Delta \mu_{2}\left(s_{n}\right)\right)^{r}
\end{aligned}
$$

If $\Psi$ is subquadratic, then the inequality sign in (19) is reversed.

Proof We start with the identity

$$
\Psi\left(A_{k} g(\mathbf{t})\right)=\Psi\left(\frac{1}{K(\mathbf{t})} \int_{\Omega_{2}} k(\mathbf{t}, \mathbf{s}) g(\mathbf{s}) \Delta \mu_{2}\left(s_{1}\right) \ldots \Delta \mu_{2}\left(s_{n}\right)\right) .
$$

By applying the refined Jensen inequality to (20), we find that

$$
\begin{aligned}
& \Psi\left(A_{k} g(\mathbf{t})\right)+\frac{1}{K(\mathbf{t})} \int_{\Omega_{2}} k(\mathbf{t}, \mathbf{s}) \Psi\left(\left|g(\mathbf{s})-A_{k} g(\mathbf{t})\right|\right) \Delta \mu_{2}\left(s_{1}\right) \ldots \Delta \mu_{2}\left(s_{n}\right) \\
& \leq \frac{1}{K(\mathbf{t})} \int_{\Omega_{2}} k(\mathbf{t}, \mathbf{s}) \Psi(g(\mathbf{s})) \Delta \mu_{2}\left(s_{1}\right) \ldots \Delta \mu_{2}\left(s_{n}\right) .
\end{aligned}
$$

Then, since $\Psi$ is nonnegative and $r \geq 1$, we have that

$$
\begin{aligned}
& \left(\Psi\left(A_{k} g(\mathbf{t})\right)+\frac{1}{K(\mathbf{t})} \int_{\Omega_{2}} k(\mathbf{t}, \mathbf{s}) \Psi\left(\left|g(\mathbf{s})-A_{k} g(\mathbf{t})\right|\right) \Delta \mu_{2}\left(s_{1}\right) \ldots \Delta \mu_{2}\left(s_{n}\right)\right)^{r} \\
& \quad \leq\left(\frac{1}{K(\mathbf{t})} \int_{\Omega_{2}} k(\mathbf{t}, \mathbf{s}) \Psi(g(\mathbf{s})) \Delta \mu_{2}\left(s_{1}\right) \ldots \Delta \mu_{2}\left(s_{n}\right)\right)^{r} .
\end{aligned}
$$

Further, by the well-known Bernoulli inequality it follows that the left-hand side of inequality (21) is not less than

$$
\Psi^{r}\left(A_{k} g(\mathbf{t})\right)+r \frac{\Psi^{r-1}\left(A_{k} g(\mathbf{t})\right)}{K(\mathbf{t})} \int_{\Omega_{2}} k(\mathbf{t}, \mathbf{s}) \Psi\left(\left|g(\mathbf{s})-A_{k} g(\mathbf{t})\right|\right) \Delta \mu_{2}\left(s_{1}\right) \ldots \Delta \mu_{2}\left(s_{n}\right),
$$

that is,

$$
\begin{aligned}
& \Psi^{r}\left(A_{k} g(\mathbf{t})\right)+r \frac{\Psi^{r-1}\left(A_{k} g(\mathbf{t})\right)}{\mathcal{K}(\mathbf{t})} \int_{\Omega_{2}} k(\mathbf{t}, \mathbf{s}) \Psi\left(\left|g(\mathbf{s})-A_{k} g(\mathbf{t})\right|\right) \Delta \mu_{2}\left(s_{1}\right) \ldots \Delta \mu_{2}\left(s_{n}\right) \\
& \quad \leq\left(\frac{1}{\mathcal{K}(\mathbf{t})} \int_{\Omega_{2}} k(\mathbf{t}, \mathbf{s}) \Psi(g(\mathbf{s})) \Delta \mu_{2}\left(s_{1}\right) \ldots \Delta \mu_{2}\left(s_{n}\right)\right)^{r} .
\end{aligned}
$$


Multiplying (23) by $\lambda(\mathbf{t})$ and integrating it over $\Omega_{1}$ with respect to $\Delta \mu_{1}\left(t_{1}\right) \ldots \Delta \mu_{1}\left(t_{n}\right)$, we have

$$
\begin{aligned}
\int_{\Omega_{1}} & \lambda(\mathbf{t}) \Psi^{r}\left(A_{k} g(\mathbf{t})\right) \Delta \mu_{1}\left(t_{1}\right) \ldots \Delta \mu_{1}\left(t_{n}\right) \\
& +r \int_{\Omega_{1}} \frac{\lambda(\mathbf{t}) \Psi^{r-1}\left(A_{k} g(\mathbf{t})\right)}{\mathcal{K}(\mathbf{t})}\left(\int_{\Omega_{2}} k(\mathbf{t}, \mathbf{s}) \Psi\left(\left|g(\mathbf{s})-A_{k} g(\mathbf{t})\right|\right) \Delta \mu_{2}\left(s_{1}\right) \ldots \Delta \mu_{2}\left(s_{n}\right)\right) \\
& \times \Delta \mu_{1}\left(t_{1}\right) \ldots \Delta \mu_{1}\left(t_{n}\right) \\
\leq & \int_{\Omega_{1}} \lambda(\mathbf{t})\left(\frac{1}{\mathcal{K}(\mathbf{t})} \int_{\Omega_{2}} k(\mathbf{t}, \mathbf{s}) \Psi(g(\mathbf{s})) \Delta \mu_{2}\left(s_{1}\right) \ldots \Delta \mu_{2}\left(s_{n}\right)\right)^{r} \\
& \times \Delta \mu_{1}\left(t_{1}\right) \ldots \Delta \mu_{1}\left(t_{n}\right) .
\end{aligned}
$$

Applying the Minkowski inequality (10) to the right-hand side of (24), we have

$$
\begin{aligned}
\int_{\Omega_{1}} \lambda(\mathbf{t})\left(\frac{1}{\mathcal{K}(\mathbf{t})} \int_{\Omega_{2}} k(\mathbf{t}, \mathbf{s}) \Psi(g(\mathbf{s})) \Delta \mu_{2}\left(s_{1}\right) \ldots \Delta \mu_{2}\left(s_{n}\right)\right)^{r} \Delta \mu_{1}\left(t_{1}\right) \ldots \Delta \mu_{1}\left(t_{n}\right) \\
\leq\left[\int_{\Omega_{2}} \Psi(g(\mathbf{s}))\left(\int_{\Omega_{1}} \lambda(\mathbf{t})\left(\frac{k(\mathbf{t}, \mathbf{s})}{\mathcal{K}(\mathbf{t})}\right)^{r} \Delta \mu_{1}\left(t_{1}\right) \ldots \Delta \mu_{1}\left(t_{n}\right)\right)^{\frac{1}{r}}\right. \\
\left.\quad \times \Delta \mu_{2}\left(s_{1}\right) \ldots \Delta \mu_{2}\left(s_{n}\right)\right]^{r}
\end{aligned}
$$

Finally, substituting (25) into (24) and taking into account definition (17) of the weight function $\eta$, we obtain that

$$
\begin{aligned}
\int_{\Omega_{1}} & \lambda(\mathbf{t}) \Psi^{r}\left(A_{k} g(\mathbf{t})\right) \Delta \mu_{1}\left(t_{1}\right) \ldots \Delta \mu_{1}\left(t_{n}\right) \\
& +r \int_{\Omega_{1}} \int_{\Omega_{2}} \lambda(\mathbf{t}) \frac{k(\mathbf{t}, \mathbf{s})}{\mathcal{K}(\mathbf{t})} \Psi^{r-1}\left(A_{k} g(\mathbf{t})\right) \Psi\left(\left|g(\mathbf{s})-A_{k} g(\mathbf{t})\right|\right) \\
& \times \Delta \mu_{1}\left(t_{1}\right) \ldots \Delta \mu_{1}\left(t_{n}\right) \Delta \mu_{2}\left(s_{1}\right) \ldots \Delta \mu_{2}\left(s_{n}\right) \\
\leq & \left(\int_{\Omega_{2}} \eta(\mathbf{s}) \Psi(g(\mathbf{s})) \Delta \mu_{2}\left(s_{1}\right) \ldots \Delta \mu_{2}\left(s_{n}\right)\right)^{r}
\end{aligned}
$$

This gives us the desired inequality (19). Similarly to the proof of (19), by the same calculations with $\Psi$ subquadratic, we see that only the inequality sign will be reversed. The proof is complete.

As a particular case of Theorem 2.1, when $\Psi(\lambda)=\lambda^{p}$ for $p \geq 2$, we have the following result.

Corollary 2.1 Let the assumptions of Theorem 2.1 be satisfied, and let $p \geq 2$. Then

$$
\begin{aligned}
& \int_{\Omega_{1}} \lambda(\mathbf{t})\left(A_{k} g(\mathbf{t})\right)^{p r} \Delta \mu_{1}\left(t_{1}\right) \ldots \Delta \mu_{1}\left(t_{n}\right) \\
& \quad+r \int_{\Omega_{1}} \int_{\Omega_{2}} \lambda(\mathbf{t}) \frac{k(\mathbf{t}, \mathbf{s})}{\mathcal{K}(\mathbf{t})}\left(A_{k} g(\mathbf{t})\right)^{p(r-1)}\left|g(\mathbf{s})-A_{k} g(\mathbf{t})\right|^{p}
\end{aligned}
$$




$$
\begin{aligned}
& \times \Delta \mu_{1}\left(t_{1}\right) \ldots \Delta \mu_{1}\left(t_{n}\right) \Delta \mu_{2}\left(s_{1}\right) \ldots \Delta \mu_{2}\left(s_{n}\right) \\
\leq & \left(\int_{\Omega_{2}} \eta(\mathbf{s}) g^{p}(\mathbf{s}) \Delta \mu_{2}\left(s_{1}\right) \ldots \Delta \mu_{2}\left(s_{n}\right)\right)^{r} .
\end{aligned}
$$

The inequality sign in (26) is reversed if $1<p \leq 2$.

Also, by choosing $\Psi:[\mathbf{a}, \infty)_{T} \rightarrow R\left(a_{i} \geq 0, i=1,2, \ldots, n\right)$ and $\Psi(\mathbf{t})=e^{\mathbf{t}}-\mathbf{t}-1$ and replacing $g(\mathbf{t})$ by $\ln g(\mathbf{t})$ in Theorem 2.1, we obtain the following multidimensional version of refined weighted Pólya-Knopp-type inequality with a kernel on time scale.

Corollary 2.2 Assume that the claims in Theorem 2.1 are satisfied, and let

$$
A_{k} g(\mathbf{t}):=\frac{1}{\mathcal{K}(\mathbf{t})} \int_{\Omega_{2}} k(\mathbf{t}, \mathbf{s}) \ln g(\mathbf{s}) \Delta \mu_{2}\left(s_{1}\right) \ldots \Delta \mu_{2}\left(s_{n}\right), \quad \mathbf{t} \in \Omega_{1} .
$$

Then

$$
\begin{gathered}
\int_{\Omega_{1}} \lambda(t)\left[\exp A_{k} g(\mathbf{t})-A_{k} g(\mathbf{t})-1\right]^{r} \Delta \mu_{1}\left(t_{1}\right) \ldots \Delta \mu_{1}\left(t_{n}\right)+I \\
\leq\left(\int_{\Omega_{2}} \eta(\mathbf{s})[g(\mathbf{s})-\ln g(\mathbf{s})-1] \Delta \mu_{2}\left(s_{1}\right) \ldots \Delta \mu_{2}\left(s_{n}\right)\right)^{r},
\end{gathered}
$$

where

$$
\begin{aligned}
I:= & r \int_{\Omega_{1}} \int_{\Omega_{2}} \lambda(\mathbf{t}) \frac{k(\mathbf{t}, \mathbf{s})}{\mathcal{K}(\mathbf{t})}\left[\exp A_{k} g(\mathbf{t})-A_{k} g(\mathbf{t})-1\right]^{r-1} \\
& \times\left[\exp \left|\ln g(\mathbf{s})-A_{k} g(\mathbf{t})\right|-\left|\ln g(\mathbf{s})-A_{k} g(\mathbf{t})\right|-1\right] \\
& \times \Delta \mu_{1}\left(t_{1}\right) \ldots \Delta \mu_{1}\left(t_{n}\right) \Delta \mu_{2}\left(s_{1}\right) \ldots \Delta \mu_{2}\left(s_{n}\right) .
\end{aligned}
$$

Remark 2.1 In Theorem 2.1, if $r=1$, then we see that the result coincides with Theorem 3.4 in [18].

In the following, we will establish some particular cases of inequalities (19) and (26) by applying Theorem 2.1 with

$$
\Omega=\Omega_{1}=\Omega_{2}=\left[a_{1}, b_{1}\right)_{T} \times\left[a_{2}, b_{2}\right)_{T} \times \cdots \times\left[a_{n}, b_{n}\right)_{T} \subset R^{n}, \quad 0 \leq a_{i}<b_{i} \leq \infty,
$$

for all $i=1,2, \ldots, n$, where $T$ is an arbitrary time scale, and the Lebesgue scale measures $\Delta \mu_{1}(\mathbf{t})=\Delta \mathbf{t}$ and $\Delta \mu_{2}(\mathbf{s})=\Delta \mathbf{s}$, and replacing $\lambda(\mathbf{t})$ by $\lambda(\mathbf{t}) / \prod_{i=1}^{n} \sigma\left(t_{i}\right)$ and $\eta(\mathbf{s})$ by $\omega(\mathbf{s}) / \prod_{i=1}^{n} s_{i}$. Then we have the following corollary.

Corollary 2.3 Let $\lambda: \Omega \rightarrow R$ be a nonnegative function, and let $k: \Omega \times \Omega \rightarrow R$ be nonnegative such that $k(\mathbf{t})$ is a $\Delta$-integrable function. Suppose that $K: \Omega \rightarrow R$ is defined by

$$
0<K(\mathbf{t}):=\int_{\Omega} k(t, s) \Delta s<\infty, \quad t \in \Omega
$$


and the function $\eta$ is defined by

$$
\omega(\mathbf{s}):=\left(\prod_{i=1}^{n} s_{i}\right)\left(\int_{\Omega}\left(\frac{k(\mathbf{t}, \mathbf{s})}{\mathcal{K}(\mathbf{t})}\right)^{r} \frac{\lambda(\mathbf{t})}{\prod_{i=1}^{n} \sigma\left(t_{i}\right)} \Delta \mathbf{t}\right)^{\frac{1}{r}}<\infty, \quad \mathbf{s} \in \Omega,
$$

where $r \geq 1$. Furthermore, assume that $g: \Omega \rightarrow R^{n}$ be $\Delta$-integrable function such that $g(\Omega) \subset R$ and define $A_{k} g: \Omega \rightarrow R$ by

$$
A_{k} g(\mathbf{t}):=\frac{1}{\mathcal{K}(\mathbf{t})} \int_{a_{1}}^{b_{1}} \ldots \int_{a_{n}}^{b_{n}} k(\mathbf{t}, \mathbf{s}) g(\mathbf{s}) \Delta \mathbf{s}
$$

If $\Psi: \Omega \rightarrow R$ is a nonnegative superquadratic function, then

$$
\begin{aligned}
\int_{a_{1}}^{b_{1}} & \ldots \int_{a_{n}}^{b_{n}} \lambda(\mathbf{t}) \Psi^{r}\left(A_{k} g(\mathbf{t})\right) \frac{\Delta \mathbf{t}}{\prod_{i=1}^{n} \sigma\left(t_{i}\right)} \\
& +r \int_{a_{1}}^{b_{1}} \ldots \int_{a_{n}}^{b_{n}} \int_{s_{1}}^{b_{1}} \ldots \int_{s_{n}}^{b_{n}} \lambda(\mathbf{t}) \Psi^{r-1}\left(A_{k} g(\mathbf{t})\right) \Psi\left(\left|g(\mathbf{s})-A_{k} g(\mathbf{t})\right|\right) \frac{\Delta \mathbf{t} \Delta \mathbf{s}}{\prod_{i=1}^{n} \sigma\left(t_{i}\right)} \\
\leq & \left(\int_{a_{1}}^{b_{1}} \ldots \int_{a_{n}}^{b_{n}} \omega(\mathbf{s}) \Psi(g(\mathbf{s})) \frac{\Delta \mathbf{s}}{\prod_{i=1}^{n} s_{i}}\right)^{r} .
\end{aligned}
$$

If $\Psi$ is subquadratic, then the inequality sign in (34) is reversed.

As a particular case of Corollary 2.3 when $\Psi(\lambda)=\lambda^{p}$ for $p \geq 2$, we have the following result.

Corollary 2.4 Let the assumptions of Corollary 2.3 be satisfied. Then

$$
\begin{aligned}
\int_{a_{1}}^{b_{1}} & \ldots \int_{a_{n}}^{b_{n}} \lambda(\mathbf{t})\left(A_{k} g(\mathbf{t})\right)^{p r} \frac{\Delta \mathbf{t}}{\prod_{i=1}^{n} \sigma\left(t_{i}\right)} \\
& +r \int_{a_{1}}^{b_{1}} \ldots \int_{a_{n}}^{b_{n}} \int_{s_{1}}^{b_{1}} \ldots \int_{s_{n}}^{b_{n}} \lambda(\mathbf{t})\left(A_{k} g(\mathbf{t})\right)^{p(r-1)}\left|g(\mathbf{s})-A_{k} g(\mathbf{t})\right|^{p} \frac{\Delta \mathbf{t} \Delta \mathbf{s}}{\prod_{i=1}^{n} \sigma\left(t_{i}\right)} \\
& \leq\left(\int_{a_{1}}^{b_{1}} \ldots \int_{a_{n}}^{b_{n}} \omega(\mathbf{s}) g^{p}(\mathbf{s}) \frac{\Delta \mathbf{s}}{\prod_{i=1}^{n} s_{i}}\right)^{r} .
\end{aligned}
$$

The inequality sign in (35) is reversed if $1<p \leq 2$.

Remark 2.2 For $r=1$, Corollaries 2.3 and 2.4 provide refinements of Theorem 3.6 and Corollary 3.7 in [5], respectively.

In the following, we present some particular inequalities with special kernels. Namely, we have the following result.

Corollary 2.5 Suppose the assumptions of Corollary 2.3 are satisfied with the kernel $k$ such that

$$
k\left(t_{1}, \ldots, t_{n}, s_{1}, \ldots, s_{n}\right):=\mathbf{0} \quad \text { if } a_{i} \leq s_{i} \leq \sigma\left(t_{i}\right) \leq b_{i}, 1 \leq i \leq n .
$$


Let $g: \Omega \rightarrow R^{n}$ be a $\Delta$-integrable function such that $g(\Omega) \subset R$, and define

$$
\begin{aligned}
\mathcal{K}(\mathbf{t}) & :=\int_{\sigma\left(t_{1}\right)}^{b_{1}} \ldots \int_{\sigma\left(t_{n}\right)}^{b_{n}} k\left(t_{1}, \ldots, t_{n}, s_{1}, \ldots, s_{n}\right) \Delta \mathbf{s}>\mathbf{0}, \quad \mathbf{t} \in \Omega, \\
\omega(\mathbf{s}) & :=\left(\prod_{i=1}^{n} s_{i}\right)\left(\int_{a_{1}}^{s_{1}} \ldots \int_{a_{n}}^{s_{n}}\left(\frac{k(\mathbf{t}, \mathbf{s})}{\mathcal{K}(\mathbf{t})}\right)^{r} \frac{\lambda(\mathbf{t})}{\prod_{i=1}^{n} \sigma\left(t_{i}\right)} \Delta \mathbf{t}\right)^{\frac{1}{r}}<\infty, \quad \mathbf{s} \in \Omega,
\end{aligned}
$$

and

$$
A_{k} g(\mathbf{t}):=\frac{1}{\mathcal{K}(\mathbf{t})} \int_{\sigma\left(t_{1}\right)}^{b_{1}} \ldots \int_{\sigma\left(t_{n}\right)}^{b_{n}} k\left(t_{1}, \ldots, t_{n}, s_{1}, \ldots, s_{n}\right) g\left(s_{1}, \ldots, s_{n}\right) \Delta \mathbf{s} .
$$

If $\Psi: \Omega \rightarrow R$ is a nonnegative superquadratic function, then inequality (34) holds. If $\Psi$ is subquadratic, then the inequality sign in (34) is reversed.

Remark 2.3 If in Corollary 2.5, we take $T=R, r=1$, and $b_{i}=\infty$ for $1 \leq i \leq n$, then we see that $\sigma\left(t_{i}\right)=t_{i}$ for $1 \leq i \leq n$, and then (34) takes the form

$$
\begin{aligned}
\int_{a_{1}}^{\infty} & \ldots \int_{a_{n}}^{\infty} \lambda\left(t_{1}, \ldots, t_{n}\right) \Psi\left(A_{k} g\left(t_{1}, \ldots, t_{n}\right)\right) \frac{d \mathbf{t}}{\mathbf{t}} \\
& +\int_{a_{1}}^{\infty} \ldots \int_{a_{n}}^{\infty}\left(\int_{s_{1}}^{\infty} \ldots \int_{s_{n}}^{\infty} \lambda(\mathbf{t}) \Psi\left(\left|g(\mathbf{s})-A_{k} g(\mathbf{t})\right|\right) \frac{d \mathbf{t}}{\mathbf{t}}\right) d \mathbf{s} \\
\leq & \int_{a_{1}}^{\infty} \ldots \int_{a_{n}}^{\infty} \omega\left(s_{1}, \ldots, s_{n}\right) \Psi\left(g\left(s_{1}, \ldots, s_{n}\right)\right) \frac{d \mathbf{s}}{\mathbf{s}}
\end{aligned}
$$

where

$$
\begin{aligned}
& \mathcal{K}(\mathbf{t}):=\int_{t_{1}}^{\infty} \ldots \int_{t_{n}}^{\infty} k\left(t_{1}, \ldots, t_{n}, s_{1}, \ldots, s_{n}\right) d \mathbf{s}>\mathbf{0}, \\
& \omega(\mathbf{s}):=s_{1} \ldots s_{n} \int_{a_{1}}^{s_{1}} \ldots \int_{a_{n}}^{s_{n}} \frac{\lambda\left(t_{1}, \ldots, t_{n}\right) k\left(t_{1}, \ldots, t_{n}, s_{1}, \ldots, s_{n}\right)}{K\left(t_{1}, \ldots, t_{n}\right)} \frac{d \mathbf{t}}{\mathbf{t}}<\infty,
\end{aligned}
$$

and

$$
A_{k} g(\mathbf{t}):=\frac{1}{\mathcal{K}(\mathbf{t})} \int_{t_{1}}^{\infty} \ldots \int_{t_{n}}^{\infty} k\left(t_{1}, \ldots, t_{n}, s_{1}, \ldots, s_{n}\right) g\left(s_{1}, \ldots, s_{n}\right) d \mathbf{s}
$$

If $\Psi$ is subquadratic, then the inequality sign in (40) is reversed. This result provides a refinement of inequality (2.5) in [19, Proposition 2.2].

Corollary 2.6 Assume that the assumptions of Corollary 2.3 are satisfied with the kernel $k$ such that

$$
k\left(t_{1}, \ldots, t_{n}, s_{1}, \ldots, s_{n}\right):=\mathbf{0} \quad \text { if } a_{i} \leq \sigma\left(t_{i}\right) \leq s_{i} \leq b_{i}, 1 \leq i \leq n .
$$

If $\Psi: \Omega \rightarrow R$ is a nonnegative superquadratic function, then inequality (34) holds for all $\Delta$-integrable functions $g: \Omega \rightarrow R^{n}$ such that $g(\Omega) \subset R$, and

$$
A_{k} g(\mathbf{t}):=\frac{1}{\mathcal{K}(\mathbf{t})} \int_{a_{1}}^{\sigma\left(t_{1}\right)} \ldots \int_{a_{n}}^{\sigma\left(t_{n}\right)} k\left(t_{1}, \ldots, t_{n}, s_{1}, \ldots, s_{n}\right) g\left(s_{1}, \ldots, s_{n}\right) \Delta \mathbf{s},
$$


where

$$
\begin{aligned}
\mathcal{K}(\mathbf{t}) & :=\int_{a_{1}}^{\sigma\left(t_{1}\right)} \ldots \int_{a_{n}}^{\sigma\left(t_{n}\right)} k\left(t_{1}, \ldots, t_{n}, s_{1}, \ldots, s_{n}\right) \Delta \mathbf{s}<\infty, \quad \mathbf{t} \in \Omega, \\
\omega(\mathbf{s}) & :=\left(\prod_{i=1}^{n} s_{i}\right)\left(\int_{s_{1}}^{b_{1}} \ldots \int_{s_{n}}^{b_{n}}\left(\frac{k(\mathbf{t}, \mathbf{s})}{\mathcal{K}(\mathbf{t})}\right)^{r} \frac{\lambda(\mathbf{t}) \Delta \mathbf{t}}{\prod_{i=1}^{n} \sigma\left(t_{i}\right)}\right)^{\frac{1}{r}}<\infty, \quad \mathbf{s} \in \Omega .
\end{aligned}
$$

If $\Psi$ is subquadratic, then the inequality sign in (34) is reversed.

Remark 2.4 If in Corollary 2.6, we take $T=R, r=1$, and $a_{i}=0$ for $1 \leq i \leq n$, then we see that $\sigma\left(t_{i}\right)=t_{i}$ for $1 \leq i \leq n$, and then (34) takes the form

$$
\begin{aligned}
\int_{0}^{b_{1}} & \ldots \int_{0}^{b_{n}} \lambda\left(t_{1}, \ldots, t_{n}\right) \Psi\left(A_{k} g\left(t_{1}, \ldots, t_{n}\right)\right) \frac{d \mathbf{t}}{\mathbf{t}} \\
& +\int_{0}^{b_{1}} \ldots \int_{0}^{b_{n}}\left(\int_{0}^{s_{1}} \ldots \int_{0}^{s_{n}} \lambda(\mathbf{t}) \Psi\left(\left|g(\mathbf{s})-A_{k} g(\mathbf{t})\right|\right) \frac{d \mathbf{t}}{\mathbf{t}}\right) d \mathbf{s} \\
\leq & \int_{0}^{b_{1}} \ldots \int_{0}^{b_{n}} \omega\left(s_{1}, \ldots, s_{n}\right) \Psi\left(g\left(s_{1}, \ldots, s_{n}\right)\right) \frac{d \mathbf{s}}{\mathbf{s}}
\end{aligned}
$$

where

$$
\begin{aligned}
& \mathcal{K}(\mathbf{t}):=\int_{0}^{t_{1}} \ldots \int_{0}^{t_{n}} k\left(t_{1}, \ldots, t_{n}, s_{1}, \ldots, s_{n}\right) d \mathbf{s}>\mathbf{0}, \\
& \omega(\mathbf{s}):=s_{1} \ldots s_{n} \int_{s_{1}}^{b_{1}} \ldots \int_{s_{n}}^{b_{n}} \frac{k\left(t_{1}, \ldots, t_{n}, s_{1}, \ldots, s_{n}\right) \lambda\left(t_{1}, \ldots, t_{n}\right)}{K\left(t_{1}, \ldots, t_{n}\right)} \frac{\mathbf{t}}{\mathbf{t}}<\infty,
\end{aligned}
$$

and

$$
A_{k} g(\mathbf{t}):=\frac{1}{\mathcal{K}(\mathbf{t})} \int_{0}^{t_{1}} \ldots \int_{0}^{t_{n}} k\left(t_{1}, \ldots, t_{n}, s_{1}, \ldots, s_{n}\right) g\left(s_{1}, \ldots, s_{n}\right) d \mathbf{s}
$$

If $\Psi$ is subquadratic, then the inequality sign in (48) is reversed. This result provides a refinement of inequality (2.2) in [19, Proposition 2.1].

Remark 2.5 For $r=1$, Corollaries 2.5 and 2.6 provide refinements of Corollaries 4.1 and 4.3 in [5], respectively.

We further present some examples. Let $K$ and $A_{k} g$ be defined as in the statements of Corollary 2.5 and use

$$
k(\mathbf{t}, \mathbf{s}):= \begin{cases}\mathbf{1}, & \text { if } a_{i} \leq s_{i} \leq \sigma\left(t_{i}\right) \leq b_{i}, 1 \leq i \leq n, \\ \mathbf{0}, & s_{i}>\sigma\left(t_{i}\right)\end{cases}
$$

Then we have the following corollary. 
Corollary 2.7 Let $\lambda: \Omega \rightarrow R$ be a nonnegative function, and let $k: \Omega \times \Omega \rightarrow R$ be nonnegative such that $k(\mathbf{t})$ is a $\Delta$-integrable function. Suppose that $\mathcal{K}: \Omega \rightarrow R$ is defined by

$$
\mathcal{K}(\mathbf{t}):=\int_{a_{1}}^{\sigma\left(t_{1}\right)} \ldots \int_{a_{n}}^{\sigma\left(t_{n}\right)} \Delta s_{1} \ldots \Delta s_{n}=\prod_{i=1}^{n}\left(\sigma\left(t_{i}\right)-a_{i}\right), \quad \mathbf{t} \in[\mathbf{a}, \mathbf{b})_{T},
$$

and let

$$
\eta(\mathbf{s}):=\left(\int_{s_{1}}^{b_{1}} \ldots \int_{s_{n}}^{b_{n}} \lambda(\mathbf{t})\left(\frac{\mathbf{1}}{\prod_{i=1}^{n}\left(\sigma\left(t_{i}\right)-a_{i}\right)}\right)^{r} \Delta \mathbf{t}\right)^{\frac{1}{r}}<\infty, \quad \mathbf{s} \in[\mathbf{a}, \mathbf{b})_{T},
$$

where $r \geq 1$. Furthermore, assume that $g: \Omega \rightarrow R^{n}$ is a $\Delta$-integrable function such that $g(\Omega) \subset R^{n}$ and $A_{k} g: \Omega \rightarrow R$ is defined by

$$
A_{k} g(\mathbf{t}):=\frac{1}{\prod_{i=1}^{n}\left(\sigma\left(t_{i}\right)-a_{i}\right)} \int_{a_{1}}^{\sigma\left(t_{1}\right)} \cdots \int_{a_{n}}^{\sigma\left(t_{n}\right)} g(\mathbf{s}) \Delta \mathbf{s} .
$$

If $\Psi: \Omega \rightarrow R$ is a nonnegative superquadratic function, then

$$
\begin{aligned}
& \int_{a_{1}}^{b_{1}} \ldots \int_{a_{n}}^{b_{n}} \lambda(\mathbf{t}) \Psi^{r}\left(A_{k} g(\mathbf{t})\right) \Delta \mathbf{t} \\
& \quad+r \int_{a_{1}}^{b_{1}} \ldots \int_{a_{n}}^{b_{n}} \int_{s_{1}}^{b_{1}} \ldots \int_{s_{n}}^{b_{n}} \lambda(\mathbf{t}) \Psi^{r-1}\left(A_{k} g(\mathbf{t})\right) \Psi\left(\left|g(\mathbf{s})-A_{k} g(\mathbf{t})\right|\right) \\
& \quad \times \frac{\Delta \mathbf{t} \Delta \mathbf{s}}{\prod_{i=1}^{n}\left(\sigma\left(t_{i}\right)-a_{i}\right)} \leq\left(\int_{a_{1}}^{b_{1}} \ldots \int_{a_{n}}^{b_{n}} \eta(\mathbf{s}) \Psi(g(\mathbf{s})) \Delta \mathbf{s}\right)^{r} .
\end{aligned}
$$

If $\Psi$ instead is subquadratic, then (56) holds in the reversed direction.

Remark 2.6 For $r=1$, inequality (56) provides a refinement of inequality (5.2) in [5, Theorem 5.1 and coincides with inequality (3.7) in [18, Corollary 3.8].

Corollary 2.8 Let the assumptions of Corollary 2.7 be satisfied, let $\lambda(\mathbf{t})$ be replaced by $\lambda(\mathbf{t}) /\left(\prod_{i=1}^{n}\left(t_{i}-a_{i}\right)\right)$, and let $\eta(\mathbf{s})$ be replaced by $\omega(\mathbf{s}) /\left(\prod_{i=1}^{n}\left(s_{i}-a_{i}\right)\right)$. Then inequality (56) reads as

$$
\begin{aligned}
& \int_{a_{1}}^{b_{1}} \ldots \int_{a_{n}}^{b_{n}} \lambda(\mathbf{t}) \Psi^{r}\left(A_{k} g(\mathbf{t})\right) \frac{\Delta \mathbf{t}}{\prod_{i=1}^{n}\left(t_{i}-a_{i}\right)} \\
& \quad+r \int_{a_{1}}^{b_{1}} \ldots \int_{a_{n}}^{b_{n}} \int_{s_{1}}^{b_{1}} \ldots \int_{s_{n}}^{b_{n}} \lambda(\mathbf{t}) \Psi^{r-1}\left(A_{k} g(\mathbf{t})\right) \Psi\left(\left|g(\mathbf{s})-A_{k} g(\mathbf{t})\right|\right) \\
& \quad \times \frac{\Delta \mathbf{t} \Delta \mathbf{s}}{\prod_{i=1}^{n}\left(t_{i}-a_{i}\right)\left(\sigma\left(t_{i}\right)-a_{i}\right)} \leq\left(\int_{a_{1}}^{b_{1}} \ldots \int_{a_{n}}^{b_{n}} \omega(\mathbf{s}) \Psi(g(\mathbf{s})) \frac{\Delta \mathbf{s}}{\prod_{i=1}^{n}\left(s_{i}-a_{i}\right)}\right)^{r},
\end{aligned}
$$

where $r \geq 1$,

$$
\omega(\mathbf{s}):=\left(\prod_{i=1}^{n}\left(s_{i}-a_{i}\right)\right)\left(\int_{[\mathbf{s}, \mathbf{b})}\left(\frac{\mathbf{1}}{\prod_{i=1}^{n}\left(\sigma\left(t_{i}\right)-a_{i}\right)}\right)^{r} \frac{\lambda(\mathbf{t})}{\prod_{i=1}^{n}\left(t_{i}-a_{i}\right)} \Delta \mathbf{t}\right)^{\frac{1}{r}}
$$

for $\mathbf{s} \in[\mathbf{a}, \mathbf{b})_{T}$, and the operator $A_{k} g$ is defined by (55). If $\Psi$ instead is subquadratic, then (58) holds in the reversed direction. 
Remark 2.7 For $r=1$, Corollary 2.8 provides a refinement of Theorem 3.1 and Remark 3.1 in [16, Theorem 3.1].

Remark 2.8 As a particular case of Corollary 2.8, when $T=R, r=1$, and $a_{i}=0$ for $1 \leq i \leq$ $n$, we see that $\sigma\left(t_{i}\right)=t_{i}$ for $1 \leq i \leq n$, and inequality (58) reads as

$$
\begin{aligned}
\int_{0}^{b_{1}} & \ldots \int_{0}^{b_{n}} \lambda(\mathbf{t}) \Psi\left(\frac{1}{\mathbf{t}} \int_{0}^{t_{1}} \ldots \int_{0}^{t_{n}} g(\mathbf{s}) d \mathbf{s}\right) \frac{d \mathbf{t}}{\mathbf{t}} \\
& +\int_{0}^{b_{1}} \ldots \int_{0}^{b_{n}} \int_{s_{1}}^{b_{1}} \ldots \int_{s_{n}}^{b_{n}} \lambda(\mathbf{t}) \Psi\left(\left|g(\mathbf{s})-\frac{1}{\mathbf{t}} \int_{0}^{t_{1}} \ldots \int_{0}^{t_{n}} g(\mathbf{s}) \mathbf{d} \mathbf{s}\right|\right) \frac{d \mathbf{t} d \mathbf{s}}{t_{1}^{2} \ldots t_{n}^{2}} \\
\leq & \int_{0}^{b_{1}} \ldots \int_{0}^{b_{n}} \omega(\mathbf{s}) \Psi(g(\mathbf{s})) \frac{d \mathbf{s}}{\mathbf{s}}
\end{aligned}
$$

where

$$
\omega(\mathbf{s}):=s_{1} \ldots s_{n} \int_{s_{1}}^{b_{1}} \ldots \int_{s_{n}}^{b_{n}} \frac{\lambda(\mathbf{t})}{t_{1}^{2} \ldots t_{n}^{2}} d \mathbf{t}<\infty, \quad \mathbf{s} \in[\mathbf{0}, \mathbf{b}) .
$$

If $\Psi$ is subquadratic, then the inequality sing in (60) is reversed, which coincides with inequality (2.2) in [20, Proposition 2.5].

Example 2.1 If we set the weight function $\lambda(\mathbf{t})=\mathbf{1}$ and $r=1$ in Corollary 2.8, then the weight function (59) yields

$$
\begin{aligned}
\omega(\mathbf{s}) & :=\left(\prod_{i=1}^{n}\left(s_{i}-a_{i}\right)\right)\left(\int_{s_{1}}^{b_{1}} \ldots \int_{s_{n}}^{b_{n}} \frac{\Delta \mathbf{t}}{\prod_{i=1}^{n}\left(t_{i}-a_{i}\right)\left(\sigma\left(t_{i}\right)-a_{i}\right)}\right) \\
& =-\left(\prod_{i=1}^{n}\left(s_{i}-a_{i}\right)\right)\left(\int_{s_{1}}^{b_{1}} \cdots \int_{s_{n}}^{b_{n}}\left(\frac{1}{\prod_{i=1}^{n}\left(t_{i}-a_{i}\right)}\right)^{\Delta} \Delta \mathbf{t}\right) \\
& =-\left(\prod_{i=1}^{n}\left(s_{i}-a_{i}\right)\right)\left(\prod_{i=1}^{n}\left(\frac{1}{b_{i}-a_{i}}-\frac{1}{s_{i}-a_{i}}\right)\right) \\
& = \begin{cases}\prod_{i=1}^{n}\left(1-\frac{s_{i}-a_{i}}{b_{i}-a_{i}}\right) & \text { if } b_{i}<\infty, 1 \leq i \leq n, \\
\mathbf{1} & \text { if } b_{i}=\infty, 1 \leq i \leq n .\end{cases}
\end{aligned}
$$

Hence inequality (58) in this setting for the case $b_{i}<\infty$ reads

$$
\begin{aligned}
\int_{a_{1}}^{b_{1}} & \ldots \int_{a_{n}}^{b_{n}} \Psi\left(A_{k} g(\mathbf{t})\right) \frac{\Delta \mathbf{t}}{\prod_{i=1}^{n}\left(t_{i}-a_{i}\right)} \\
& \quad+\int_{a_{1}}^{b_{1}} \ldots \int_{a_{n}}^{b_{n}} \int_{s_{1}}^{b_{1}} \ldots \int_{s_{n}}^{b_{n}} \Psi\left(\left|g(\mathbf{s})-A_{k} g(\mathbf{t})\right|\right) \frac{\Delta \mathbf{t} \Delta \mathbf{s}}{\prod_{i=1}^{n}\left(t_{i}-a_{i}\right)\left(\sigma\left(t_{i}\right)-a_{i}\right)} \\
\leq & \int_{a_{1}}^{b_{1}} \ldots \int_{a_{n}}^{b_{n}} \prod_{i=1}^{n}\left(1-\frac{s_{i}-a_{i}}{b_{i}-a_{i}}\right) \Psi(g(\mathbf{s})) \frac{\Delta \mathbf{s}}{\left(s_{i}-a_{i}\right)},
\end{aligned}
$$


whereas the case $b_{i}=\infty$ yields

$$
\begin{aligned}
\int_{a_{1}}^{b_{1}} & \ldots \int_{a_{n}}^{b_{n}} \Psi\left(A_{k} g(\mathbf{t})\right) \frac{\Delta \mathbf{t}}{\prod_{i=1}^{n}\left(t_{i}-a_{i}\right)} \\
& \quad+\int_{a_{1}}^{b_{1}} \ldots \int_{a_{n}}^{b_{n}} \int_{s_{1}}^{b_{1}} \ldots \int_{s_{n}}^{b_{n}} \Psi\left(\left|g(\mathbf{s})-A_{k} g(\mathbf{t})\right|\right) \frac{\Delta \mathbf{t} \Delta \mathbf{s}}{\prod_{i=1}^{n}\left(t_{i}-a_{i}\right)\left(\sigma\left(t_{i}\right)-a_{i}\right)} \\
\leq & \int_{a_{1}}^{b_{1}} \ldots \int_{a_{n}}^{b_{n}} \prod_{i=1}^{n} \Psi(g(\mathbf{s})) \frac{\Delta \mathbf{s}}{\left(s_{i}-a_{i}\right)},
\end{aligned}
$$

where the operator $A_{k} g$ is defined by (55). If $\Psi$ instead is subquadratic, then the inequality sign in (62) and (63) is reversed.

Remark 2.9 Inequalities (62) and (63) provide a refinement of the results in [16, Example 3.1]. For $a_{i}=0$ for $1 \leq i \leq n$, they provide a refinement of the results in [5, Corollary 5.3, Example 5.4].

Remark 2.10 If we set $n=1$, then Example 2.1 provides a refinement of Corollary 2.1 in [21, Corollary 2.1]. Also, in the particular case $n=2$, inequality (62) provides a refinement of Theorem 3.2 in [21, Corollary 2.1].

Remark 2.11 As a particular case of Example 2.1, when $T=R$ and $a_{i}=0$ for $1 \leq i \leq n$, we see that $\sigma\left(t_{i}\right)=t_{i}$ for $1 \leq i \leq n$, and so (62) reads as

$$
\begin{aligned}
\int_{0}^{b_{1}} & \ldots \int_{0}^{b_{n}} \Psi\left(\frac{1}{t_{1} \ldots t_{n}} \int_{0}^{t_{1}} \ldots \int_{0}^{t_{n}} g(\mathbf{s}) d \mathbf{s}\right) \frac{d \mathbf{t}}{t_{1} \ldots t_{n}} \\
& +\int_{0}^{b_{1}} \ldots \int_{0}^{b_{n}} \int_{s_{1}}^{b_{1}} \ldots \int_{s_{n}}^{b_{n}} \Psi\left(\left|g(\mathbf{s})-\frac{1}{t_{1} \ldots t_{n}} \int_{0}^{t_{1}} \ldots \int_{0}^{t_{n}} g(\mathbf{s}) d \mathbf{s}\right|\right) \frac{d \mathbf{t} d \mathbf{s}}{t_{1}^{2} \ldots t_{n}^{2}} \\
\leq & \int_{0}^{b_{1}} \ldots \int_{0}^{b_{n}} \prod_{i=1}^{n}\left(1-\frac{s_{i}}{b_{i}}\right) \Psi(g(\mathbf{s})) \frac{d \mathbf{s}}{s_{1} \ldots s_{n}}
\end{aligned}
$$

which coincides with inequality (2.3) in [20, Remark 2.6]. In the one-dimensional case $(n=1)$, this reduces to Example 4.1 in [17]. Inequality (62) yields

$$
\begin{aligned}
\int_{0}^{b_{1}} & \ldots \int_{0}^{b_{n}} \Psi\left(\frac{1}{t_{1} \ldots t_{n}} \int_{0}^{t_{1}} \ldots \int_{0}^{t_{n}} g(\mathbf{s}) d \mathbf{s}\right) \frac{d \mathbf{t}}{t_{1} \ldots t_{n}} \\
& +\int_{0}^{b_{1}} \ldots \int_{0}^{b_{n}} \int_{s_{1}}^{b_{1}} \ldots \int_{s_{n}}^{b_{n}} \Psi\left(\left|g(\mathbf{s})-\frac{1}{t_{1} \ldots t_{n}} \int_{0}^{t_{1}} \ldots \int_{0}^{t_{n}} g(\mathbf{s}) d \mathbf{s}\right|\right) \frac{d \mathbf{t}}{t_{1}^{2} \ldots t_{n}^{2}} d \mathbf{s} \\
\leq & \int_{0}^{b_{1}} \ldots \int_{0}^{b_{n}} \Psi(g(\mathbf{s})) \frac{d s_{1} \ldots d s_{n}}{s_{1} \ldots s_{n}} .
\end{aligned}
$$

Now we further consider some generalizations of Pólya-Knopp-type inequalities.

Corollary 2.9 Assume that the assumptions of Corollary 2.7 be satisfied with $n=1, a_{1}=$ $a \geq 0, r \geq 1$, and $b_{1}=\infty$. If $\Psi: I \rightarrow R$ is a nonnegative superquadratic function, where 
$I \subset R$ is an interval, then

$$
\begin{aligned}
& \int_{a}^{\infty} \lambda(t) \Psi^{r}\left(\frac{1}{\sigma(t)-a} \int_{a}^{\sigma(t)} g(s) \Delta s\right) \Delta t \\
& \quad+r \int_{a}^{\infty} \int_{s}^{\infty} \lambda(t) \Psi^{r-1}\left(\frac{1}{\sigma(t)-a} \int_{a}^{\sigma(t)} g(s) \Delta s\right) \\
& \quad \times \Psi\left(\left|g(\mathbf{s})-\left(\frac{1}{\sigma(t)-a} \int_{a}^{\sigma(t)} g(s) \Delta s\right)\right|\right) \frac{\Delta t}{\sigma(t)-a} \Delta s \\
& \leq\left(\int_{a}^{\infty}\left(\int_{s}^{\infty} \lambda(t)\left(\frac{1}{\sigma(t)-a}\right)^{r} \Delta t\right)^{\frac{1}{r}} \Psi(g(s)) \Delta s\right)^{r}
\end{aligned}
$$

for all $\Delta$-integrable functions $g: \Omega \rightarrow R$ such that $g(\Omega) \subset I$. If $\Psi$ instead is subquadratic, then (66) holds in the reversed direction.

Example 2.2 In addition to the assumptions of Corollary 2.9, if $T$ consists of only isolated points, then (66) takes the form

$$
\begin{aligned}
& \sum_{t \in[a, \infty)_{T}} \lambda(t) \Psi^{r}\left(\frac{1}{\sigma(t)-a} \sum_{s \in[a, \sigma(t))_{T}} g(s)(\sigma(s)-s)\right)(\sigma(t)-t) \\
& \quad+r \sum_{s \in[a, \infty)_{T}}\left(\sum_{t \in[s, \infty)_{T}} \lambda(t) \Psi^{r-1}\left(\frac{1}{\sigma(t)-a} \sum_{s \in[a, \sigma(t))_{T}} g(s)(\sigma(s)-s)\right)\right. \\
& \left.\quad \times \Psi\left(\left|g(s)-\frac{1}{\sigma(t)-a} \sum_{s \in[a, \sigma(t))_{T}} g(s)(\sigma(s)-s)\right|\right) \frac{\sigma(t)-t}{\sigma(t)-a}\right)(\sigma(s)-s) \\
& \leq\left(\sum_{s \in[a, \infty)_{T}}\left(\sum_{t \in[s, \infty)_{T}} \lambda(t)\left(\frac{1}{\sigma(t)-a}\right)^{r}(\sigma(t)-t)\right)^{\frac{1}{r}} \Psi(g(s))(\sigma(s)-s)\right)^{r} .
\end{aligned}
$$

Remark 2.12 For $r=1$, Corollary 2.7 and Example 2.2 provide refinements of Corollary 5.7 and Example 5.8 in [5], respectively. They also are a refinement of Theorem 1.1 in [29], but here we use time scales notation instead of the notation given in [29].

Remark 2.13 As in Example 2.2, we can write a discrete version of (56) and (58).

Acknowledgements

The authors would like to thank the referees for their important comments and remarks.

\section{Funding}

Not applicable.

\section{Availability of data and materials}

Not applicable.

\section{Competing interests}

The authors declare that they have no competing interests.

\section{Authors' contributions}

The authors contributed equally to the writing of this paper. All authors approved the final version of the manuscript.

\section{Author details}

${ }^{1}$ Department of Mathematics, Faculty of Science, Mansoura University, Mansoura, Egypt. ${ }^{2}$ Department of Mathematics, Faculty of Science, Al-Azhar University, Nasr City, Egypt. ${ }^{3}$ College of Engineering, Applied Science University, Manama, Kingdom of Bahrain. ${ }^{4}$ Department of Mathematics, Cankaya University, Ankara, Turkey. ${ }^{5}$ Institute of Space Sciences,

Magurele-Bucharest, Romania. 


\section{Publisher's Note}

Springer Nature remains neutral with regard to jurisdictional claims in published maps and institutional affiliations.

\section{Received: 14 May 2019 Accepted: 18 November 2019 Published online: 03 December 2019}

\section{References}

1. Abramovich, S., Banić, S., Matić, M.: Superquadratic functions in several variables. J. Math. Anal. Appl. 137(2), $1444-1460(2007)$

2. Abramovich, S., Jameson, G., Sinnamon, G.: Inequalities for averages of convex and superquadratic functions. J. Inequal. Pure Appl. Math. 7(2), Art. 70 (2004)

3. Agarwal, R.P., O'Regan, D., Saker, S.H.: Hardy Type Inequalities on Time Scales. Springer, Cham (2016)

4. Barić, J., Bibi, R., Bohner, M., Pečarić, J.: Time scales integral inequalities for superquadratic functions. J. Korean Math. Soc. 50, 465-477 (2013)

5. Bohner, M., Nosheen, A., Pečarić, J., Younis, A.: Some dynamic Hardy-type inequalities with general kernels. Math. Inequal. Appl. 8, 185-199 (2014)

6. Bohner, M., Peterson, A.: Dynamic Equations on Time Scales: An Introduction with Applications. Birkhäuser, Boston (2001)

7. Bohner, M., Peterson, A. (eds.): Advances in Dynamic Equations on Time Scales. Birkhäuser, Boston (2003)

8. Čižmešija, A., Pečarić, J., Persson, L.E.: On strengthened Hardy and Pólya-Knopp's inequalities. J. Approx. Theory 125 74-84 (2003)

9. Gao, F.: General fractional calculus in non-singular power-law kernel applied to model anomalous diffusion phenomena in heat transfer problems. Therm. Sci. 21, 11-18 (2017)

10. Godunova, E.K.: Generalization of a two-parameter Hilbert inequality. Izv. Vysš. Učebn. Zaved., Mat. 54(1), 35-39 (1967) (Russian)

11. Golmankhaneh, A.K., Fernandez, A.: Random variables and stable distributions on fractal Cantor sets. Fractal Fract. 3, 31 (2019)

12. Golmankhaneh, A.K., Fernandez, A., Baleanu, D.: Diffusion on middle- $\xi$ Cantor sets. Entropy 20, 504 (2018)

13. Hardy, G.H.: Notes on some points in the integral calculus, LX. An inequality between integrals. Messenger Math. 54 150-156 (1925)

14. Kaijser, S., Persson, L.E., Öberg, A.: On Carleman and Knopp's inequalities. J. Approx. Theory 117, 140-151 (2002)

15. Knopp, K.: Über Reihen mit positiven Gliedern. J. Lond. Math. Soc. 3, 205-311 (1928)

16. Oguntuase, J.A.: Hardy type inequalities on time scales. Publ. Inst. Math. 98(112), 219-226 (2015)

17. Oguntuase, J.A., Persson, L.E.: Refinement of Hardy's inequalities via superquadratic and subquadratic functions. J. Math. Anal. Appl. 339, 1305-1312 (2008)

18. Oguntuase, J.A., Persson, L.E.: Time scales Hardy-type inequalities via superquadraticity. Ann. Funct. Anal. 5(2), 61-73 (2014)

19. Oguntuase, J.A., Persson, L.E., Essel, E.K.: Multidimensional Hardy type inequalities with general kernels. J. Math. Anal. Appl. 348(1), 411-418 (2008)

20. Oguntuase, J.A., Persson, L.E., Essel, E.K., Popoola, B.A.: Refined multidimensional Hardy type inequalities via superquadraticity. Banach J. Math. Anal. 2, 129-139 (2008)

21. Özkan, U.M., Yildirim, H.: Hardy-Knopp type inequalities on time scales. Dyn. Syst. Appl. 17, 477-486 (2008)

22. Özkan, U.M., Yildirim, H.: Time scale Hardy-Knopp type integral inequalities. Commun. Math. Anal. 6, 36-41 (2009)

23. Parvate, A., Gangal, A.D.: Calculus on fractal subsets of real-line l: formulation. Fractals 17, 53-148 (2009)

24. Saker, S.H.: Lyapunov inequalities for half-linear dynamic equations on time scales and disconjugacy. Dyn. Contin. Discrete Impuls. Syst., Ser. B, Appl. Algorithms 18, 149-161 (2011)

25. Saker, S.H.: Applications of Opial and Wirtinger inequalities on zeros of third order differential equations. Dyn. Syst. Appl. 20, 479-494 (2011)

26. Saker, S.H.: Lyapunov type inequalities for a second order differential equations with a damping term. Ann. Pol. Math. 103, 37-57 (2012)

27. Saker, S.H.: Opial's type inequalities on time scales and some applications. Ann. Pol. Math. 104, 243-260 (2012)

28. Sulaiman, T.A., Yavuz, M., Bulut, H., Baskonuse, H.M.: Investigation of the fractional coupled viscous Burgers' equation involving Mittag-Leffler kernel. Phys. A, Stat. Mech. Appl. 527, 121126 (2019)

29. Vasić, P.M., Pečarić, J.: Notes on some inequalities for convex functions. Mat. Vesn. 6 (19)(2), 185-193 (1982)

30. Yang, X.: On a Liapunov-type inequality for a certain higher-order differential equations. Appl. Math. Comput. 134, 307-317 (2003)

31. Yang, X.J.: General Fractional Derivatives: Theory, Methods and Applications. CRC Press, New York (2019)

32. Yang, X.J., Ragulskis, M., Taha, T.: A new general fractional-order derivative with Rabotnov fractional-exponential kernel applied to model the anomalous heat transfer. Therm. Sci. 23(3 Part A), 1677-1681 (2019). https://doi.org/10.2298/TSCl180320239Y 